\title{
The Secrets of Cancer Spontaneous Resolution Lie in the Working Mode of the Immune System
}

Jianqing $\mathrm{Wu}^{1}$, Ph.D., J.D. and Ping Zha, M.D. (Chi. Med.)

Correspondence: tempaddr2@atozpatent.com

Draft dated August 9, 2021, updated August 11, 2021

1. Healthier World, P. O. Box 689, Beltsville, MD 20704. www.igoosa.com.

Keywords: human immune system dynamics, immune functional fluctuations, multiple factor health optimization, reductionist (?) limitations, incurable diseases, cancer and COVID-19

\section{INTRODUCTION}

The spontaneous resolution of cancer is known for hundreds to potentially thousands of years. It is now accepted as an indisputable fact. There are hundreds of documented cases and perhaps millions of undocumented cases [a reasonable hypothesis]. Past literature demonstrates that cancer regression is usually associated with acute infections, fever, and immunostimulation. More recent study found that cancer can be prevented by major changes of lifestyle. However, medicine uses chemotherapy and surgeries as the primary treatments even though the cancer somatic mutation theory is largely irrelevant to most cancer cases and the performance of those measures is generally poor. A large number of recent studies show that cancer is a disease aggravated by pollutants, chronic stress, and fault lifestyle.

Cancer miracles imply that cancer must be curable and preventable. Wu's two decades of research and our recent efforts give us the answer. Human immune system is an emotional creature that is run and controlled by emotional, environment, activities, nutrition, etc. This is a matter that affects most individu- 
als and most families, we urge research community to explore this new approach.

\section{PIONEER STUDIES TO CHALLENGE MEDICAL MODELS}

In 2018, we wrote about cancer after Dr. Wu have done years of researches on cancer. Cancer deeply affects Dr. Wu. A large number of his family members, friends, coworkers, and people close to him have lost their lives. We saw a large number of cancer patients in the last a few years. In 2001, by strike of luck, Dr. $\mathrm{Wu}$ healed his three-decade hip joint pain by doing mind exercise, and that success completely changed his belief on chronic diseases including cancer. He believed that predictable cures for chronic diseases would be much simpler.

Finding real problems in medicine is entirely a different thing. For a strange reason, $\mathrm{Dr}$. $\mathrm{Wu}$ found part the answer from his legal career. Many of rules are presumed to be correct without actually proving they are in fact correct. He quickly narrowly down his focus to the presumptions used in medical research and treatment models. However, he lacked data to prove anything for years. In the last a few years, medical researches suddenly started producing more and more evidence that $\mathrm{Dr}$. Wu was waiting. In 2019, we posed our first article on the flaws in using clinical trials for studying chronic diseases. In this study, we used a unique method of proof: we constructed two kinds of trials by assuming effects of a single factor treatment and a multiple factors treatment by using the same assumptions that would routinely be used in medical research, we then conducted a mock research to see if the research model could correctly determine the assumed treatment effects. It cannot. We traced the problem to the statistical model, and found it attempts to use statistical model to solve a problem that could never be solved. One root problem is that the model attempts to bundle all weak factors into the experimental error. This is clearly wrong because if statistical analysis can have this magic power, we do not need to develop instrument to increase detection limit for studying weak factors.

This error from using statistical analysis is fatal in studying chronic diseases. The true effect of any weak factor cannot be determined as long as there is at least one weak factor interfering with the weak factor. If a researcher measures the total benefits of $\mathrm{N}$ factors like a bundled treatment, the treatment statistics would be enlarged by $\mathrm{k}^{*} \sqrt{ } \mathrm{k}$, where $\mathrm{k}$ is the number of weak factors with a similar degree of effect. If the total number of weak factors is increased from 1 to $2,5,10$, and 100 , the treatment statistics would increase by $2.8,11.2,32$, and 
1000 times. This means that bundling weak effects as an error is improper for studies that require HIGH accuracy. In reality, we found that the departure from important biological pathways may be as little as $0.1 \%-1 \%$ for most chronic diseases. It takes years to develop most diseases like cancer. This slow-developing nature means that studies of chronic disease require much higher accuracy or separation of all influencing factors.

We also found that mathematical operation is misused to get an averaging effect of weak factors. For example, the nutritional status of any nutrient and a toxic substance is unique in each person. If a treatment for correcting malnutrition or a detoxifying a substance is randomly applied to all people in a treatment group, its beneficial effects on some patients can be "nullified" by its negative or adverse effects on others. Thus, fifty negative responses and fifty positive responses may become zero mean. This does not mean the treatment has no effects at all. It means that the treatment benefits some people and also poisons some people. This also means that a negative finding in a clinical trial does not guarantee it is actually harmful to ALL people. What is told is that such a treatment is harmful to more people than it is beneficial. The outcome simply means that the treatment mean happens to be on the negative side, as compared with the control. Even if a same life factor is NOT used in the control group, this factor can still have a positive or negative effect on each patient. For example, a particular infectious agent may appear in some patients and miss in others; each toxic substance can exist in different amounts in different persons.

Health optimization is completely different from a randomized trial. All controllable factors are used as part of the treatment and are used only on the persons who need them. We predict that the treatment of using 100 factors could deliver 100 times of a single factor (assuming each of them has the same amount of benefit). Population medicine made an assumption that all people are similar. As far as disease causes for chronic diseases are concerned, people cannot be treated as same or similar to apples, but as a mixture of oranges, apples, sesames, and water melons, etc. For other research purposes like evaluating housing, food allowances, etc., all people may be treated as apples.

We further found that "When the apparent "error" is far too large, relative to the effects of the treatment, data set tends to come out with test statistics falling on the region of acceptance of the null hypothesis, thus resulting in false acceptance of the null hypothesis or false rejection of true treatment effects. Those fatal flaws are expected to exist under most circumstances. No statistical method, no any other methods under the Sun, can ever correct this great bias that arises from breaching the core presumption used in the statistical model. 
Thus, clinical trials are invalid and have been misused in studying chronic diseases."

In our second study [2], we deeply examined the presumptions that are used in the medical research and treatment models and we found that all key presumptions do not hold. We further found that statistical observations do not exist even though they are widely used in medical researches (except for studying a few things like body weight and height). Observations must be values representing a property that is fully defined and cannot be changed during a drawing trial. In a clinical trial, data could be freely swapped between any two members within a treatment group. Swapping data between Patient X and Patient $\mathrm{Y}$ within a treatment would not make a difference. In the worst scenario, a $20 \%$ effectiveness rate could be found on two completely different groups of people. Saving these 20 patients or saving those 20 patients have same meaning in clinical trials, but have dramatically different consequences to patients. Thus, an identical conclusion at the same probability level means completely different things to different persons. By focusing on one single conclusion with a $\mathrm{P}$ level, the population research model is unable to address personal differences. Naturally, treatments from a best population outcome often do not work for specific persons.

To further explore the flaws in clinical trials, we found, among others, that statistical population of health properties simply does not exist for most research purposes, mathematical models are misused to model intensive properties, and reductionist treatments are inferior and inherently dangerous [2]. One big flaw is use of mathematical operations to sum up data from different systems (e.g., different persons). For numbers to be additive, they must be in the same unit, scale, and have similar significance. One class of properties is intensive property that reflects the physical property of a system. Temperature at one system and temperature at another system are not additive because heat represented by any temperature range are different, and temperature at DIFFERENT SYSTEMS such as water and gas means completely different things. They cannot be added up to get an average. All health properties (except weights and volumes) are similar to intensive properties that must be tightly confined to the system (e.g., the person). Medicine made the biggest mistake since the start of using clinical trials almost three centuries ago, and the mistake becomes worse after the method is used to study chronic diseases.

The flaw of cross-system modeling can be seen by considering a car model. A Civic uses fuel at the rate of 1 gallon/time and an Accord, at the rate of 2 gallons/time. Their average, 1.5 gallons/time, may indicate the average fuel usage, 
which is useful in determining fuel supply and demand. However, this number cannot be used to force both cars to run at the average rate. Each of the two values is firmly associated with the particular car and its specific circumstances. The average has no meaning if it is viewed out of the context. We found, for example, that average of organ function between a 180-lb man and a 90-lb woman has no meaning because the average can be applied to neither the man nor the woman. If the average is forced on both of them, the imposed function would be insufficient to run the big man, but will blow out the little lady. We found that all disease properties cannot be summed and averaged among different persons. While studies involving averaging personal data may be useful for OTHER purposes including studying strong treatment effects, they cannot be used as the basis for validating or rejecting a treatment for chronic diseases.

In our third study, we extensively examined the organ functional changes in a person's life time, and found that a person's lifespan would highly depend on the life stress that burdens vital organs. A person may feel no symptom if the stress does not deplete the organ's function to below $40 \%$. Moreover, it is the common knowledge that a vast number of toxic substances including heavy metals, natural compounds, industrial chemicals, therapeutic drugs, etc. do not cause any discomfort during exposure. Yet, they can cause severe damages to organs and ruin personal health. As shown in FIG. 3 below, a person has a usable organ capacity of $100 \%$, he may need only about $40 \%$ or less to maintain essential functions (the line indicated by basal functional capacity), while the death threshold is even lower (may be about 20\%-30\%). Any life stress such as diseases, drugs, toxic substances, and intensive exercises can temporarily depress the organ's functions. It then recovers with loss of some capacity in some cases. Due to this huge organ functional buffer, research conclusions based on symptoms (rather than the detailed alternations of cells) cannot be trusted.

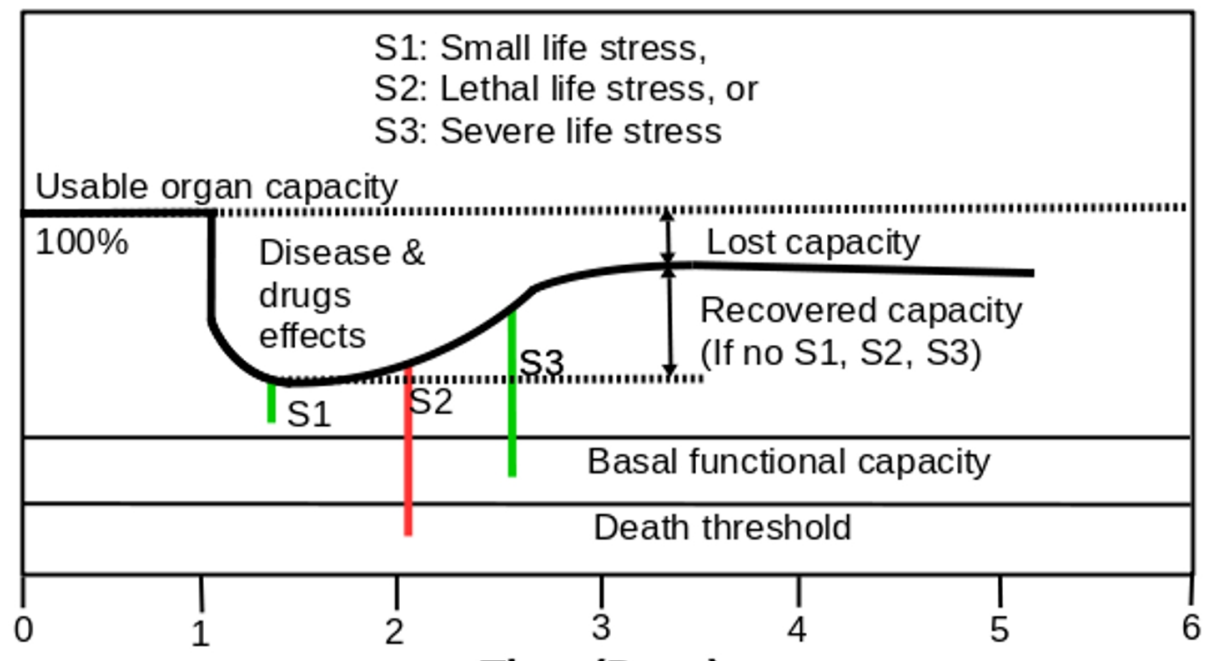

Time (Days) 
The flaws in researches are well reflected in massive product liability cases concerning substances such as DES, asbestos, Agent Orange, DDT, Roundup, "forever chemicals", etc. In case of Roundup, 800 studies done in nearly 40 years still failed to predict its side effects. Based on court reports on product liability cases, clinical trials and population studies always fail to predict long-term side effects. This is not strange because subtle damages are done to reduce the organ functional capacity (e.g., organ functional reserve). In addition, side effects are often in different forms while studies could not focus on all possible forms of side effects. Motivation is one big thing: a sponsor of a drug has no duty to prove no side effect, but the consumers do not have resources to study. It is a common pattern that consumers will make a case for removing dangerous products by showing injuries, disabilities, and deaths. Indeed, abundant cases implies that resolution of symptoms does not guarantee that the exposed substance do not cause permanent damages to the user, but the real question is the size of damage.

\section{THE CONCEPT: THE DANCING OF IMMUNITY}

Based on our previous studies, we found medical researches are incapable of accurately determining the true effects of life factors. We want to prove how weak factors such as lifestyle, dietary, emotional, disease agents, and environmental factors actually affect personal health and chronic diseases.

\section{A. Existing Evidence}

While tens of thousands of studies have provided irrefutable evidence to support our multiple disease causes model, which blurs differences between causal factors, associated factors, and risk factors, etc.

Our mathematical simulations show that the actual affects of $\mathrm{N}$ factors are at least the sum of all weak factors when each is matched to the patient but not randomly used. 
Our presumption analysis reveals that averaging of data from different person is also flawed. All people are different as far as their health and disease conditions are concerned.

In this study, we explore the immune system's dynamics and show that how personal life factors affect personal health and disease outcomes, and why their effects can be added.

\section{B. Our Approach}

We expand the scope of the immune functions to include functions for removing garbage and repairing tissue damages, and then identify six classes of health properties for evaluating the immune system's actual functional capabilities. The immune system is supposed to correct any fault in any part of the body, including peripheral tissues and tissues in any vital organ. In this model, personal ability to resist infection depends on the immune system's current functional capabilities; and the ability to resolve existing diseases depends on the immune system's actual functional capabilities integrated over a relevant time. We show how each life factor affects the immune system's current functional capabilities by examining its effects on any of the six classes of properties.

Based on the six classes of properties, we can predict that a good immune system must have (1) a good capillary network: a high density of capillaries (more capillaries per unit cross-section area) and extensive distribution of capillaries in the tissue; (2) large capillaries pores (lack of fats deposit); (3) healthy cells and ordered organization or high elasticity of the tissue; (4) a good blood composition (the counts of red blood cells, white blood cells and applets are in the right values); (5) lack of in-plasma toxic substances that can interfere with biological and cellular processes; and (6) a proper (inherent) blood flow rate or local blood pressure. In such a tissue, immune cells can migrate into the tissue, perform required immune function fast, and may move out of the tissue quickly. Based on our method for analyzing the life factors on the six classes of health property of tissues, we then can predict how the immune system's functional capacities change in each daily cycle.

\section{The Findings}

Based on massive factual discoveries from published articles, we list at least sixteen classes of life factors that can affect both immune function capacities by current impacts and permanent impacts. They include (1) exposure to low temperature; (2) prolonged intensive exercise; (3) severe emotional (lasting chronic stress, extreme emotional distress, and excessive worries); (4) trauma, accidents, and wounds; (5) toxic substances at toxicity levels in plasma; (6) toxic 
substances without noticeable symptoms; (7) acute allergic reactions; (8) food poisoning; (9) environmental factors (temperature, humidity, oxygen level, vibrations, etc); (10) other infections; (11) malnutrition and temporary nutritional imbalance; (12) sleep deprivation or oversleep; (13) fatigue and exhaustion; (14) alcohol, recreational drugs, smoking, etc.; (15) medications (immune suppressants, pain killers, cancer drugs etc.); (16) other chronic diseases; (17) A combination of various immune suppressive factors and events. The permanent total impacts caused by all those factors are shown in FIG. 2 (the difference between Line A and B, and the effects of several exemplar factors are shown in the checkshaped curves. The magnitudes of loss would depend on age, personal lifestyle, exposure to toxic substances, etc.

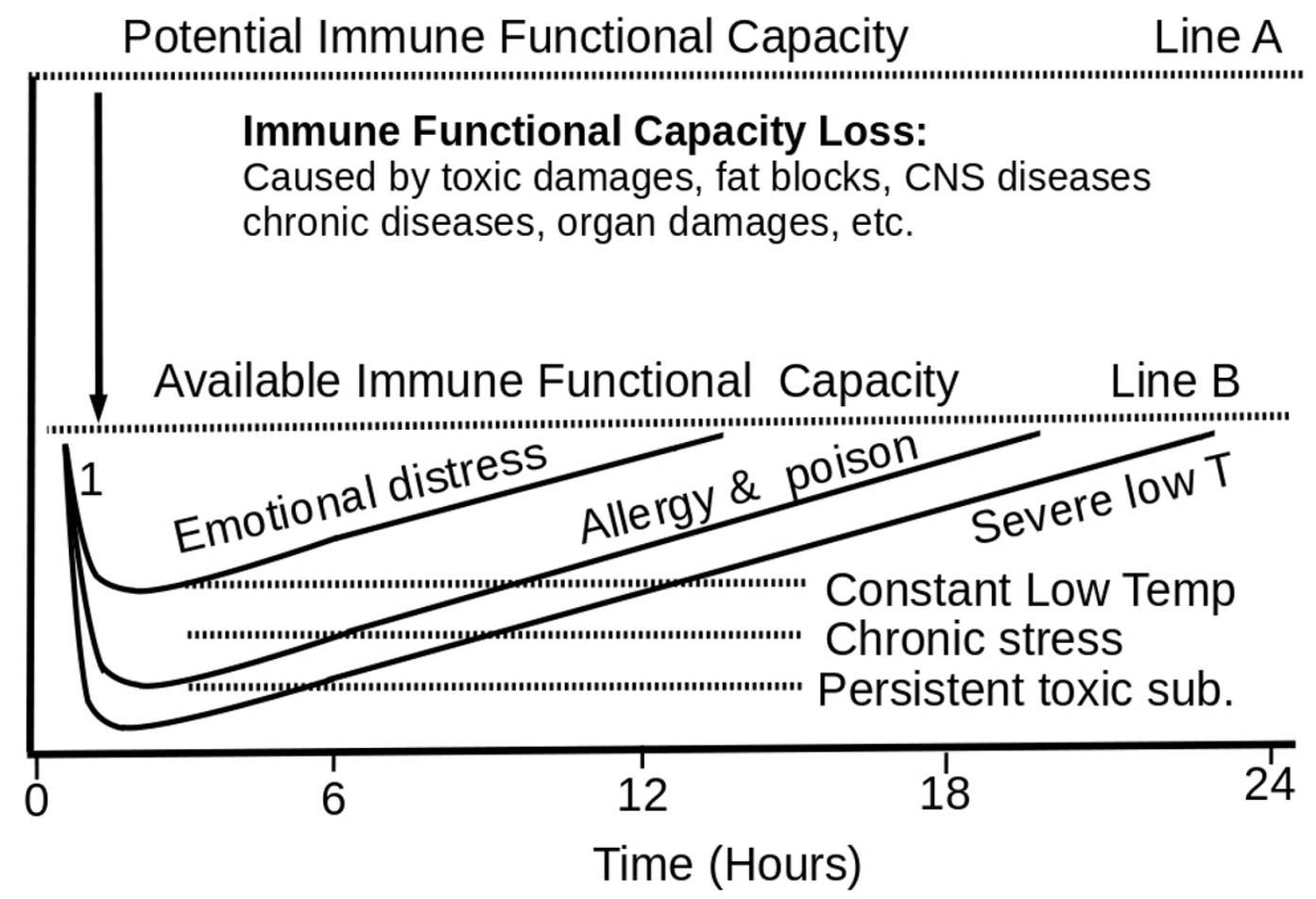

A person has the maximum immune functional capacities indicated by Line A. Due to aging and cell damages, the person will have reduced maximum immune functional capacities, as indicated by line $\mathrm{B}$. The value indicated by $\mathrm{B}$ is only a potential maximum value that may be unavailable but is unavailable due to other reasons. Most of the potential functional capabilities are further suppressed by any of current effects of those listed factors and their combinations. 
The immune functional capacities depend on several critical functions such as garbage removal, tissue repair, and tissue remodeling, and require support of liver, lungs, heart, and kidneys, which are shared global biological resources. If any of those functions fails, the immune system's functional capacities must be severely depressed or temporarily lost.

The sizes of impacts of the immune suppressive factors are implied in the following facts: (1) temperature could alter local blood flow rate by up to 20 times; (2) exercise could change cancer survival rates of many types of cancer for various survival years by nearly $40 \%$; (3) personal body position could change internal local blood pressure inside the brain by more than $50 \mathrm{~mm} \mathrm{Hg}$ pressure (one meter water high is $73 \mathrm{~mm} \mathrm{Hg}$ ); (4) warming foot by a warm foot bath can raise systemic blood flow rate by many times at reduced heart work; (5) most drugs' current impacts could be more than 10-20 times of their lasting baseline impacts (referred from their half lives); (6) food poisoning and subtle toxic impacts must be very large (based on severe symptoms); (7) a demand triggered by any factor could generate 20 to $80 \mathrm{~mm} \mathrm{Hg}$ additional burden to the heart (a rough estimate based on pulse pressure), and (8) a bad argument in a wrong environment and wrong time may be a game-end mistake (a common factor for triggering stroke). Sometimes, one single factor may have the power of checkmate in ruining personal health.

Based on the above findings, we constructed a hypothetical immune functional capacity diagram for a hypothetical person in FIG. 3 below.

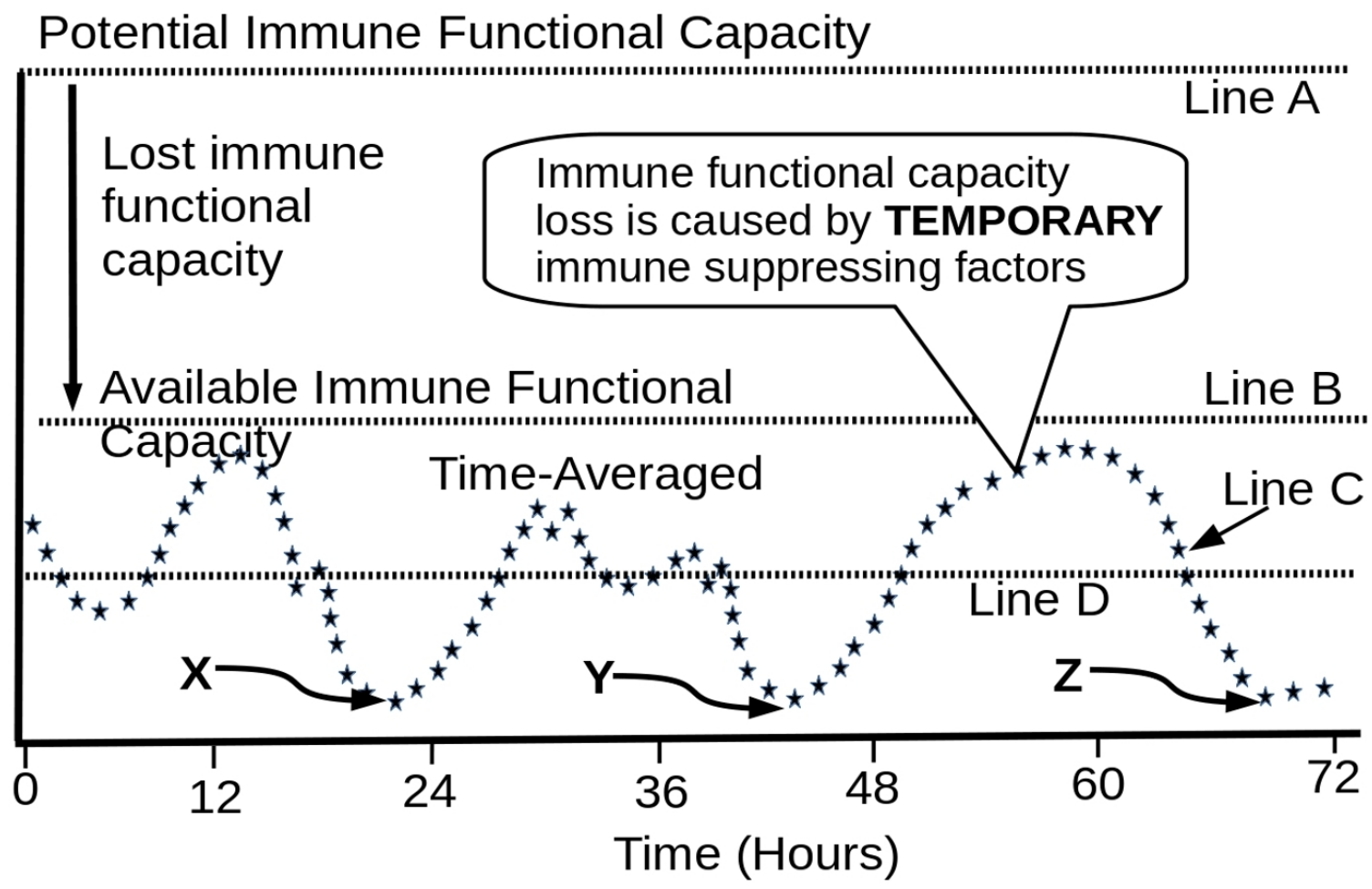


Since each of the vital functions is influenced by both current and permanent effects of a large number of life factors, the effects of all influencing factors are naturally additive (most probably in non-linear manners). If each of 100 substances can affect the tissue organization by current toxic effects, 100 substances must be much worse than a single factor. This is one reason that they are additive.

Effects of most life factors are additive through shared vital organ functions. The overall capabilities of garbage removal and tissue repairing functions may be roughly expressed as time-averaged current functional capabilities over a relevant time period. If wastes are produced by infection A, toxic substance reaction $B$, and intensive exercise $C$, then the total waste accumulating speed would depend on all of them. Thus, whether the tissue can recover from a viral infection A would depend on how much waste is produced by other causes such as toxic substance reaction B and exercise C. Therefore, toxic substances and intensive exercise burdens become a factor for determining final disease outcome.

Based on indications of kinetic data of a large number of substances and things, we anticipate that the overall immune capabilities may be only $30 \%-80 \%$ of the maximum values. It is even possible the immune system is too tired to fight. We can safely assume there are HUGE rooms for boosting the current functional capacities, and there are fair rooms for enlarging the potential immune functional capacities (line A) and organ's functional capacities.

The effects of many life factors can also be explained by their accumulative effects over time. All biological processes operate on a continuous basis. The life factors affect the health or disease state by influencing all biological processes over the entire time period. All historical effects of all life factors are recorded in the tissue. All relevant variables such as toxic substance numbers, their relative concentrations, and their historical effects are reflected in the historical damages in tissues. Indeed, cancer is caused by only a small imbalance in some biological processes. Now, a patient has $\mathrm{N}$ cause-related factors and $\mathrm{M}$ seemingly unrelated factors to use in fighting any chronic disease. Any claim that chronic diseases are incurable by nature cannot be correct. The concept of using two sets of life factors is applicable to cancer, heart diseases, AIDS, COVID-19, rare diseases, etc.

Many seemingly irrelevant factors can affect cancer outcomes because they can have different burdens on the shared biological resources for removing 
garbage, repairing tissue damages and remodeling tissues. Based on all the reasons, we found that rational strategies for conquering cancer are identifying disease causes, avoiding exposure to disease agents, detoxifying toxic substances, and using seemingly-irrelevant life factors to boost real-time immune functional capabilities and vital organ functions. All cancers are influenced by $\mathrm{N}$ and $\mathrm{M}$ factors, where $\mathrm{N}$ represents the number of factors related to cancer causes, and $\mathrm{M}$ represents the number of factors that influence the immune functional capacities and organ capacities. Most of $\mathrm{N}$ factors are pollutants (in the broadest sense) while most of $\mathrm{M}$ factors are social practices that are formed after the Industrial Revolution, particularly the modern era.

For prevention of cancer, one needs to avoid all toxic substances as much as possible. The second class of factors are the stress from daily life which is responsible for switching on human stress hormones for months and years, with predictable adverse effects on human health. The third class of factors is completely harmless and often appears to be irrelevant. (The fourth factors, mindbody interaction is outside the scope of this article).

\section{A NOVEL APPROACH TO FIGHTING CANCER}

We will discuss how to use our findings to conquer cancer. For cancer to develop on a person, the person must have exposed to one or more causecausing agents (causal factors), the body must have lost the anti-tumor immunity, and in addition, a large number of seemingly unrelated factors also aggravate the disease by influencing organ functions and suppressing the immune system. Those factors have suppressed the anti-tumor immunity. The immune cells could not move into the diseased tissue, lack required energy to perform their functions, are chemically interfered by certain toxic substances, could not remove wastes from killed cells, and/or have a diminished capacity to repair existing damages in tissue.

While various toxic substances (more than the carcinogens) play an important role in initiation of cancer, we must find that emotion can be a very important factor influencing disease outcomes. We know two instances that tell the important role of "Voodoo spell". In one case, an old may was diagnosed with a final stage of stomach cancer. He sought a best surgeon to have the cancer removed. He was told that the operation was highly successful. To most people who known his case, his cancer had spread to other organs such as the liver and the whole abdomen and the surgeon could not do anything other than stitching 
the opening back. The man trusted his surgeon, retired from his job, and made some changes to his lifestyle. More than thirty years later, he was still alive with no sign of getting a cancer. In another case, an old man died one day before his negative cancer diagnosis report was issued. Before his death, the person had suspected that he had cancer. When a person knows that he has a cancer, he normally experience very high chronic stress which completely alters his stress hormone pattern. It may have a big impact on his daily immune functional capacities. In contrast to the first case, the patient got substantial benefits of "reversed" Voodoo spell.

If we look at the immune capacity dancing diagram FIG. 3. The stress has an effect to cause the person to constantly pump stress hormones. If one understands our full analysis, one should see that cancer is much easier to conquer than it appears to be. Many studies imply that a complete change of lifestyle will be the cure and prevention. What we would like to add is that a change of lifestyle is intended to disrupt certain family habits and activity patterns that are associated with exposures to cancer agents. But a similarly important thing is that one must, among others, get rid of the Voodoo spell. While it is difficult, but one needs to learn devices to stop the thought that cancer is a terminal disease. It is terminal only if one believes in it and thus turns his stress hormones on for months and years, severely suppressing the antitumor immunity.

Our final point is that no chronic disease can be cured by drugs, but always be healed and cured by the immune system. Medicine can render help with urgent matter that immune system could not due to the acute nature. We believe all spontaneous resolutions are not spontaneous. Those winners might have done enough to change their lifestyle and personal activities, without knowing their benefits, to make their tissue environments unfit for cancer proliferation. This short article is intended to shed light on how to fight cancer. The life art to successfully conquer cancer lies in boosting the working mode of the immune system for all time. If one truly believes it, he is about to win half a battle before the start. However, one should never hope to win by doing very little or nothing. One still needs to overcome kinetic barriers for self-resolution and the statemaintaining roles of the Central Nervous System. Every patient needs to fight in the right way with a firm resolve to win.

\section{OUR RESEARCHES}


1. Wu J, Zha P. Randomized Clinical Trial Is Biased and Invalid In Studying Chronic Diseases, Compared with Multiple Factors Optimization Trial. Preprints 2019, 2019110245. Accessed from https://www.preprints.org/manuscript/ 201911.0245/v1. DOI: http://dx.doi.org/10.2139/ssrn.3480523

2. Wu J, Zha P. Clinical Trials and Reductionist Approach Preclude Cures for Chronic Diseases Due to Flawed Presumptions. Preprints 2020, 2020090572 (doi: 10.20944/preprints202009.0572.v1). Accessed from https://www.preprints.org/manuscript/202009.0572/v1

3. Wu J, Zha P. A Multi-Factor Model for Estimating Relative Lifespans and Extending Health Spans. Accessed from https://papers.ssrn.com/sol3/papers.cfm?abstract_id=3502432

4. Wu J, Zha P. Immune Functional Capability Dynamics Determine Disease Outcomes as in Cancer, Heart Diseases, COVID-19, Etc. https://osf.io/skf8e. 